\title{
Author Correction: The clinical impact of glycobiology: targeting selectins, Siglecs and mammalian glycans
}

Benjamin A. H. Smith (D) and Carolyn R. Bertozzi

Correction to: Nature Reviews Drug Discovery https://doi.org/10.1038/s41573-020-00093-1, published online 18 January 2021.

In a glossary term, glycosylation was stated to occur on the wrong amino acid. This has been corrected in the online version.

https://doi.org/10.1038/s41573-021-00160-1 I Published online 8 February 2021

๑) Springer Nature Limited 2021 